\title{
Diagnostics of a high pressure DC magnetron argon discharge with an Aluminium Cathode
}

\author{
S. Mitic, ${ }^{1, *}$ J. Moreno, ${ }^{2}$ C. Arnas,${ }^{3}$ and L. Couëdel ${ }^{2,3, \dagger}$ \\ ${ }^{1}$ Justus-Liebig-University, Giessen, Germany \\ ${ }^{2}$ Department of Physics and Engineering Physics, \\ University of Saskatchewan, Saskatoon, SK S7N 5E2, Canada \\ ${ }^{3}$ CNRS, Aix-Marseille Université, PIIM, 13397 Marseille, France
}

(Dated: June 25, 2021) 


\begin{abstract}
In this article, the plasma parameters of a direct-current magnetron discharge in argon at moderate pressure (10 Pa to $40 \mathrm{~Pa}$ ) using an aluminium cathode are explored. The density of argon excited states, the sputtered aluminium atom density and the electron temperature are estimated using a collisional radiative model. The electron temperature obtained using optical emission spectroscopic data agrees well with measurements made using a Langmuir probe. The influence of the discharge parameters, namely the background argon pressure and the discharge current are discussed.
\end{abstract}

\title{
I. INTRODUCTION
}

Magnetron discharges are widely used for thin film deposition [1]. In planar magnetron discharges, magnetic fields created by permanent magnets positioned behind the cathode, trap electrons near the cathode region allowing plasma production at relatively low pressures $[2,3]$, therefore favouring cathode material sputtering by energetic ions [4]. For the deposition of metallic coating (copper, aluminium, tungsten, etc), inert gases such as argon (Ar) or krypton (Kr) are usually used [1, 5-8].

Nanoparticle (NP) formation in glow discharges has been the subject of many studies during the past decades. For example, NP growth and effects on discharge parameters have been studied in radio-frequency $(\mathrm{RF})$ discharges using reactive gases such as silane [9] or acetylene [10], and by sputtering of the electrodes $[11,12]$. Studies have also been devoted to the study of the growth and the dynamics of the NP clouds in direct-current (DC) sputtering glow discharges using different cathode materials [13-16]. It was shown that the growth process strongly depends on the type of cathode material and other discharge parameters, such as the neutral gas pressure and the discharge current. In the specific case of the formation of tungsten NPs, it was shown that the formation mechanisms and transport in between electrodes exhibit complex dynamics [17]. Since the mid-1990's, RF and DC magnetron sputtering in gas aggregation sources (MS-GAS) is commonly used to produce metal NPs [18-26]. In most cases, MS-GAS consists of a high-pressure chamber in which

* slobodan.mitic@exp1.physik.uni-giessen.de

† lenaic.couedel@usask.ca, lenaic.couedel@univ-amu.fr 
the magnetron discharge is located connected to a low pressure expansion chamber in which MS-GAS from the atoms ejected by sputtering of the magnetron cathode. However, even though MS-GAS are extensively used to produce NPs of many different materials, very few studies are devoted to the correlation of NP growth dynamics in the high pressure chamber with the parameters of the magnetron discharges $[24,27,28]$. The formation of NPs in a DC planar magnetron discharge in argon at relatively high pressures (10s of $\mathrm{Pa})$ is also possible $[29,30]$. In particular experimental studies on the formation of tungsten NPs in high pressure DC planar magnetron argon discharges and their influence on plasma parameters were recently done [31-33]. Nevertheless DC magnetron plasmas in high pressure regimes are still poorly understood.

In this article, the dependance of the plasma parameters on the discharge conditions in a conventional DC magnetron set up were investigated. The cathode was made of aluminium $(\mathrm{Al})$ and the background gas was Ar. The pressure $\left(p_{\mathrm{Ar}}\right)$ was varied as well as the discharge current $\left(I_{\mathrm{d}}\right)$. The current-voltage relations of the magnetron plasma were estblished for different $p_{\mathrm{Ar}}$. In addition, optical emission spectroscopy (OES) measurements on Ar I and Al I lines were taken. A collisional radiative model including self-absorption corrections was used to find the argon metastable atom density, aluminium atom density, electron temperature $T_{\mathrm{e}}$ and the electron density $n_{\mathrm{e}}$. Finally, the different plasma parameters $\left(n_{\mathrm{e}}\right.$, $T_{\mathrm{e}}$ and plasma potential $V_{p}$ ) were also measured at a fixed distance under the cathode on the symmetric axis of the magnetron discharge using a cylindrical Langmuir probe. Plasma parameters obtained with probe and OES measurements were compared and the different results are discussed.

\section{EXPERIMENTAL SET-UP}

The experiments were performed using a planar unbalanced DC magnetron source (mcseROBEKO) in Ar. The aluminium cathode had a diameter of 3 inches and was facing a grounded anode $10 \mathrm{~cm}$ below its surface. A grounded guard-ring of $2 \mathrm{~cm}$ width and $1 \mathrm{~cm}$ height and $\sim 7.4 \mathrm{~cm}$ inner diameter was positioned under and around the cathode assembly at a distance of $0.2 \mathrm{~cm}$ from the aluminium surface (see Fig.1). Two glass half-cylinders were used to confine the plasma (and the produced nanoparticles). In order to perform 


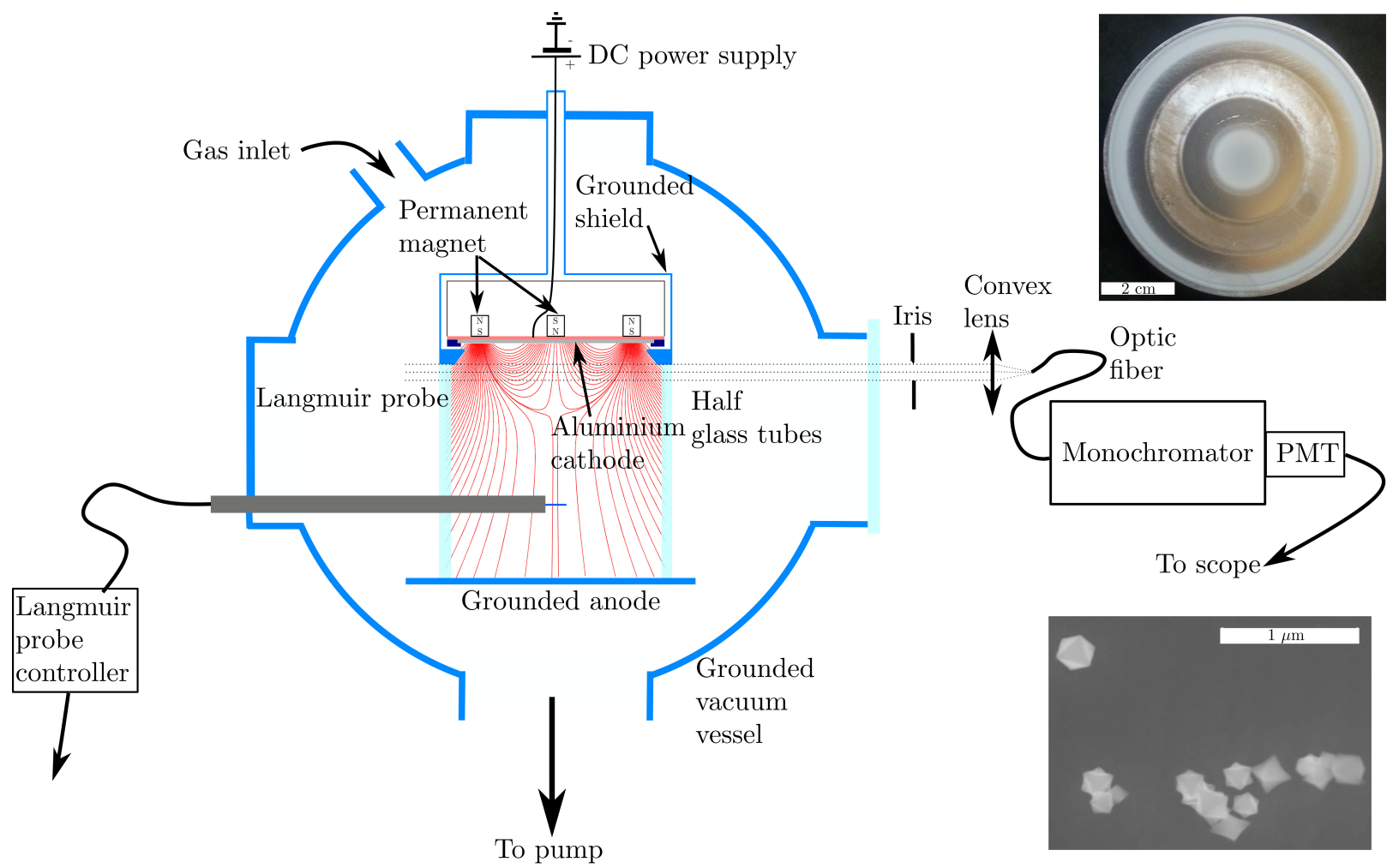

FIG. 1. (Colour online) Schematic of the experimental setup. The red lines illustrate the magnetic field lines. The top right inset shows the state of aluminium cathode after the experiments. Note the circular sputtering track. The bottowm righ inset shows a scanning electron microscope image of aluminium nanoparticle grown in a plasma with $I_{\mathrm{d}}=300 \mathrm{~mA}$ at $p_{\mathrm{Ar}}=30 \mathrm{~Pa}$ for a plasma duration of $60 \mathrm{~s}$.

optical emission spectroscopy and radial Langmuir probe measurements, a $1 \mathrm{~cm}$ gap was kept between them.

The discharge assembly was installed in a stainless steel cylinder device of $40 \mathrm{~cm}$ length and $30 \mathrm{~cm}$ diameter. The base vacuum was maintained at $\leq 10^{-6}$ mbar using a turbo molecular pump (Edwards TurboVac 400). An argon pressure between $10 \mathrm{~Pa}$ and $40 \mathrm{~Pa}$ at a flow of $5 \mathrm{sccm}$ gas was set during the experiments. A current-regulated DC power supply (Glassman HV, $1 \mathrm{~A}-1 \mathrm{kV}$ ) was used to bias the cathode and maintain the discharge current at a constant value (from $100 \mathrm{~mA}$ to $500 \mathrm{~mA}$ ). The cathode was actively cooled with a flow of $\sim 1 \mathrm{~L} / \mathrm{min}$ of water at room temperature. Before each series of experiments, the cathode was cleaned by of low pressure $\left(\sim 10^{-1} \mathrm{~Pa}\right)$, high current $(>0.5 \mathrm{~A})$ plasma pulses. Under the chosen operating conditions, the cathode was sputtered and aluminium nanoparticles 
could be grown (see bottom right inset of Fig.1). A precise description of these particles and

\section{ELECTRICAL CHARACTERISTICS OF THE DC MAGNETRON DISCHARGE}

The current-voltage relation in planar magnetrons can provide indirect information about the sputtering rate in the magnetron as it strongly depends on the background gas pressure and the dynamics of the sputtered cathode atoms [36]. Note that current-voltage relationships also depend on the gas-target combination, the secondary electron emission rate due to ion impact, the design of the magnetron, the magnetic field strength, and other experimental parameters [37]. For this reason, the current-voltage relation was measured for four pressures, for currents varying between $100 \mathrm{~mA}$ and $600 \mathrm{~mA}$. The results are shown in Fig. 2 . As can be seen, all measured $I_{d}-V_{c}$ characteristics follow a curve that obeys the relation:

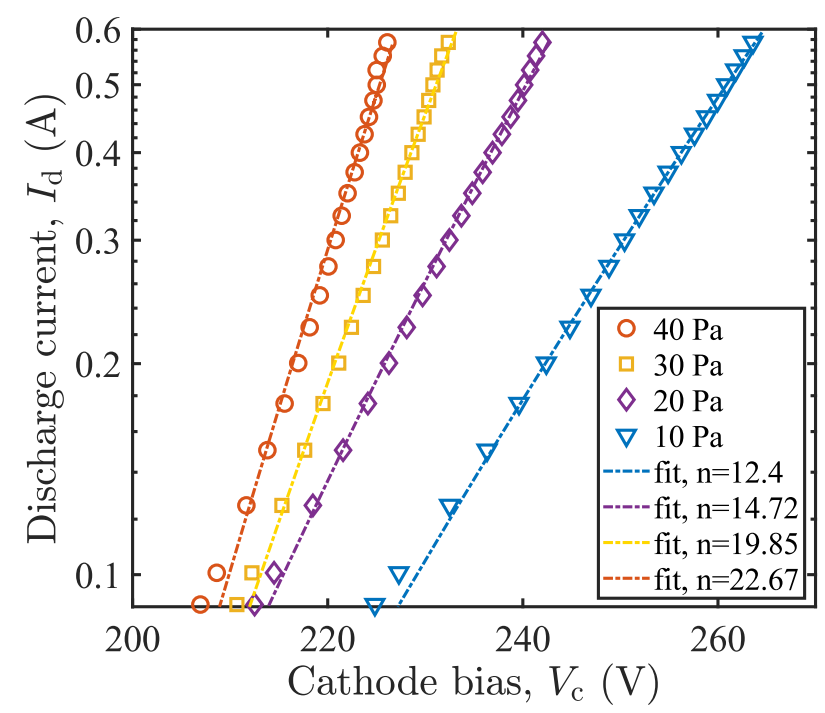

FIG. 2. (Colour online) Discharge current $I_{d}$ as a function of the cathode voltage $V_{c}$ for different argon pressures. Each $I_{\mathrm{d}}-V_{\mathrm{c}}$ curve is fitted by a power law curve of the form $I_{\mathrm{d}}=k \cdot V_{\mathrm{c}}^{n}$. The value of $n$ obtained for each pressure is given in the inset.

$$
I_{d}=k \cdot V_{\mathrm{c}}^{n}
$$


When increasing the pressure from $10 \mathrm{~Pa}$ to $40 \mathrm{~Pa}$, the exponent $n$ increases from $n \simeq 12$ high pressures than at low pressures. Since collisions with the neutral background are more frequent at high pressures, the secondary electrons emitted by the cathode due to ion and fast neutral bombardment with a non-zero energy can be recaptured by the cathode due to gyration around the magnetic field lines [38, 39]

\section{LANGMUIR PROBE MEASUREMENTS}

\section{A. Experimental set-up}

A cylindrical Langmuir probe (tungsten filament, $300 \mu \mathrm{m}$ in diameter, $11.28 \mathrm{~mm}$ long) was used to measured plasma parameters $\sim 3 \mathrm{~cm}$ above the anode on the discharge axis (see Fig. 1). This corresponds to a region where the magnetic field is sufficiently low to consider the electrons unmagnetised. Moreover the magnetic field lines are perpendicular to the probe axis, allowing us to use standard probe analysis to recover the plasma parameters. Finally being about $7 \mathrm{~cm}$ away from the cathode allowed us to make the measurements in a region where the number density of nanoparticles is low [28] and where the aluminium coating of the probe assembly develops slowly enough so the probe characteristics remain unaffected for the duration of the experiment.

The measurements were performed in a steady state discharge (constant cathode bias and plasma emission intensity). Moreover, additional laser light scattering experiments seemed to indicate that there were indeed no trapped particles in the probed region. To obtain the current-voltage $(I V)$ characteristics, the probe voltage $V_{\mathrm{p}}$ was ramped up from $-35 \mathrm{~V}$ to $+10 \mathrm{~V}$ by step of $0.33 \mathrm{~V}$. Depending on the discharge parameters, the probe current $I_{\mathrm{p}}$ varied from $\sim-100 \mu \mathrm{A}$ to $\sim+35 \mathrm{~mA}$. Each measurement was repeated 3 times and averaged out to obtain the $I_{\mathrm{p}}-V_{\mathrm{p}}$ curves.

\section{B. Results}

Due to the high sputtering rate of aluminium, the probe was rapidly coated by an aluminium layer rendering a study over the range of parameters accessible to optical emission spectroscopy measurements very complicated. The Langmuir probe measurements were 
(a)

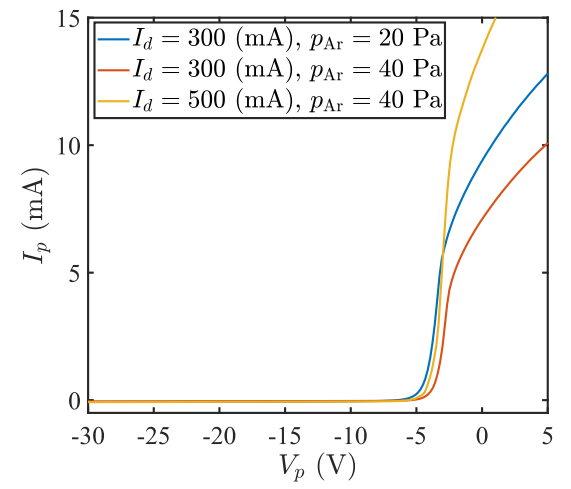

(b)

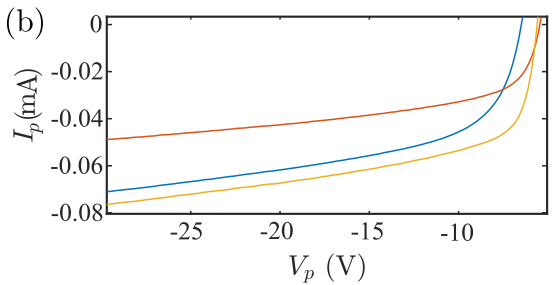

(c)

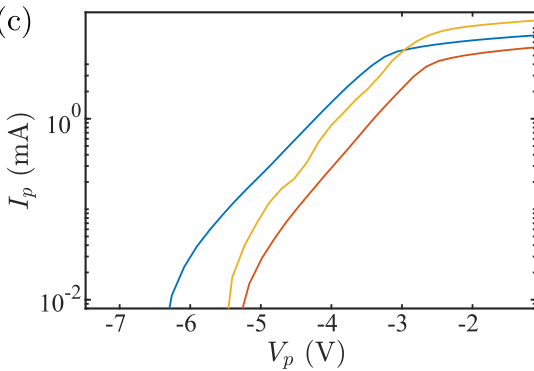

FIG. 3. (a) Raw $I V$ curves for different discharge parameters. (b) Zoom on the ion part. (c) Zoom on the transition part in semi-log scale.

therefore limited to a smaller set of currents and pressures: $I_{\mathrm{d}}=300 \mathrm{~mA}$ and $I_{\mathrm{d}}=500 \mathrm{~mA}$ for pressures between $20 \mathrm{~Pa}$ and $40 \mathrm{~Pa}$.

In Fig. 3, raw Langmuir probe $I V$ characteristics are shown for different conditions. Analysis showed that the electron energy distribution functions were quasi-Maxwellian (see Fig. 3(c) and inset of Fig. 4(b)). The analyses of the Langmuir probe $I V$ characteristics were performed using both the Langmuir analysis [40] and Druyvesteyn analysis [41] which allowed us to recover the different $n_{e}$ and $T_{e}$ values. The two methods gave very close results and the average values of $n_{e}$ and $T_{e}$ for the investigated discharge parameters are shown in Fig. 4. Note that the measurements being made far away from the cathode where the electrons are confined by the magnetic field, the reported values of electron density are at least one to two orders of magnitude lower than that in the plasma ring confined by the magnetic arch near the cathode. Moreover, the electrons are colder by about $\sim 1 \mathrm{eV}[24,33]$. Consequently only the trends are of interest in this study.

As expected, a higher current results in a higher plasma density (Fig. 4(a)). With $n_{\mathrm{e}} \sim$ $2.5 \cdot 10^{10} \mathrm{~cm}^{-3}$ at $I_{\mathrm{d}}=300 \mathrm{~mA}$ and $n_{\mathrm{e}} \sim 5 \cdot 10^{10} \mathrm{~cm}^{-3}$ at $I_{\mathrm{d}}=500 \mathrm{~mA}$, the values of electron density are in agreement with those observed in experiments using a tungsten cathode [33] or using a titanium cathode [24] with similar discharge currents and background gas pressures. The plasma density is only slightly dependant on the gas pressure and even show a small 

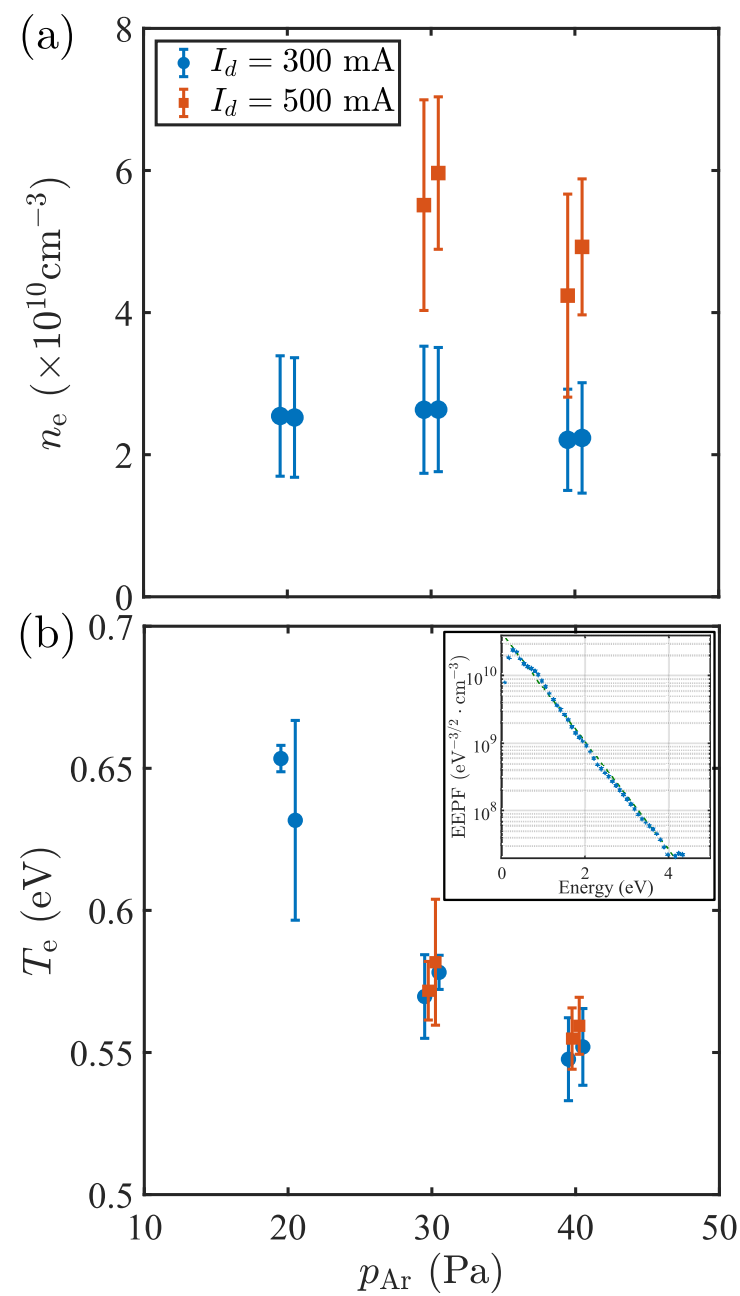

FIG. 4. (a) Electron density, $n_{\mathrm{e}}$. (b) Electron temperature, $T_{\mathrm{e}}$. The inset is the electron energy probability function (EEPF) obtained using Druyvesteyn analysis on the Langmuir probe $I V$ characteristics measured at $p_{\mathrm{Ar}}=40 \mathrm{~Pa}$ and $I_{\mathrm{d}}=300 \mathrm{~mA}$. In both (a) and (b), the presented values are the average of the the Langmuir and Druyvesteyn analyses $\left(T_{e}\right.$ is obtained by a fit of the EEPF by a Maxwellian EEPF for the Druyvesteyn analysis). Note that the points have been artificially scattered around their true pressure value for clarity of the plot.

decrease when increasing the background gas pressure at constant discharge current. Increasing the pressure results in a decrease of the electron temperature (from $0.65 \mathrm{eV}$ down to $0.55 \mathrm{eV}$, see Fig. 4(b)). The electron temperature seems, however, almost insensitive to the discharge current. The measured electron temperatures are lower than the ones measured in the same experiment using a tungsten cathode $[32,33]$ but close to the one measured under similar experimental conditions with a titanium cathode [24]. 


\section{OPTICAL EMISSION SPECTROSCOPY}

\section{A. Experimental set-up}

OES studies of the discharge plasma were carried out using a monochromator (Acton Spectra Pro 2500i, $500 \mathrm{~mm}$ focal length) coupled to a photomultiplier tube (Hamamatsu R3896). Convex lenses and an optic fibre were used to collect the emitted plasma radiation, $\sim 1.5 \mathrm{~cm}$ below the aluminium cathode where the line of sight crosses the high density plasma ring below the cathode (see Fig. 1). A previous study with a tungsten cathode showed that nanoparticles could be trapped on the edge of the last magnetic arch $(\sim 4 \mathrm{~cm}$ below the cathode) [33] and the line of sight used for the optical spectrum acquisition was considered free of large nanoparticles [42]. The spectra were then calibrated in intensity using a spectral radiance calibrated SphereOptics SR-3A integration sphere placed in front of the light collection system (glass window, convex lenses, optic fibre, monochromator, and photomultiplier tube). After calibration, the relative intensities of each spectral line could be compared.

\section{B. Collisional radiative model of an argon discharge containing aluminium}

Plasma parameters were evaluated based on the optical emission spectra recorded in the range from 300 to $830 \mathrm{~nm}$ consisting of a set of most prominent argon lines in the red and near infra-red range as well as a few strong Al transition lines close to the UV range, as visible in Fig. 5.

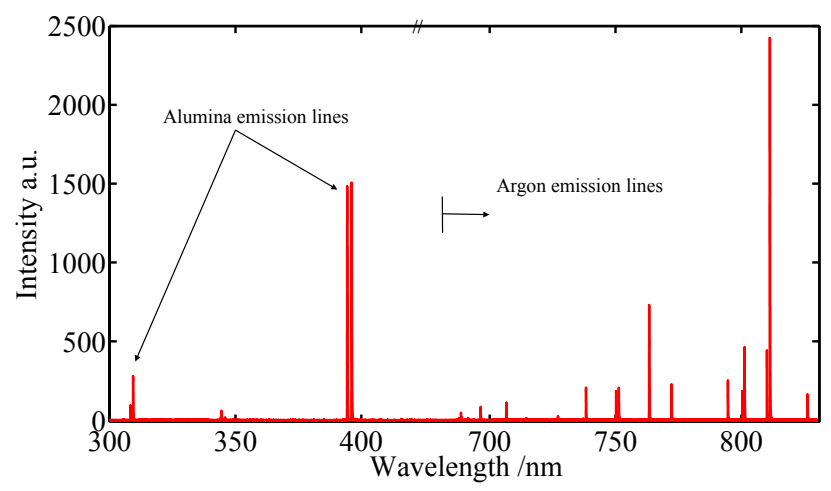

FIG. 5. (Colour online) Example of the measured emission spectra. 
Emission spectra of an argon low temperature plasma have been successfully analysed by $[43,44]$. In the complete model for argon $[44,45]$ the populations of the first 14 excited states $(\operatorname{Ar}(1 \mathrm{~s})$ and $\operatorname{Ar}(2 \mathrm{p})$ branches) were modelled including electron impact excitation from ground state, electron coupling between and within the $\operatorname{Ar}(1 \mathrm{~s})$ and $\operatorname{Ar}(2 \mathrm{p})$ levels, and electron quenching and radiative processes including light trapping. It was shown that in the case of low temperature plasmas with moderate electron densities and temperatures, the collisional-radiative model ( $\mathrm{CRM})$ can be simplified to an extended corona model including excitation from ground and 1 s states and radiation transfer processes [44, 46]. As an outcome of such a description, the population of $\operatorname{Ar}(2 \mathrm{p})$ excited states linearly depends on the electron density making the analysis only dependent on the electron temperature.

In this case, the rate balance equation for excited $\operatorname{Ar}\left(2 \mathrm{p}_{\mathrm{i}}\right)$ state can be written as:

$$
\frac{d n_{2 p i}}{d t}=\frac{N \cdot K_{g-2 p i}\left(T_{e}\right)+\sum_{j=2.5} n_{1 s j} \cdot K_{1 s j-2 p i}\left(T_{e}\right)}{\sum_{j=2 . .5} A_{i j} \cdot \gamma\left(1 s_{j}\right)}
$$

where $N$ is the argon ground state density, $\mathrm{n}_{1 \mathrm{sj}}$ is the $\operatorname{Ar}(1 \mathrm{~s})$ state densities, $\mathrm{K}_{\mathrm{g}-2 \mathrm{pi}}$ and $K_{1 s j-2 p i}$ are rate coefficients for electron impact excitation of $\operatorname{Ar}\left(2 p_{i}\right)$ from ground and $\operatorname{Ar}(1 \mathrm{~s})$ states respectively. Radiative processes are described by Einstein coefficients for spontaneous emission $\mathrm{A}_{\mathrm{ij}}$ corrected by radiation trapping (self-absorption) factor $\gamma(1 s j)[47,48]$.

Similar to our previous works $[44,46]$ the argon spectra was first analysed using the line branching method in order to estimate the argon 1s state densities that play an important role in the whole CRM and are non-trivial to model. The line branching model compares measured intensities of lines originating from the same excited level, to the theoretically estimated ratios (ratios of the Einstein coefficients for spontaneous emission), as in [44, 49]. The difference can be further refined by a self-absorption mechanism [50] depending on the densities of the lower state of the considered transitions. In such a case observing two sets of lines transitioning to the same two lower levels (e.g. $\operatorname{Ar}(1 \mathrm{~s} 4)$ and $\operatorname{Ar}(1 \mathrm{~s} 5)$ ) would lead to the evaluation of the lower state densities. In the case of Ar emission spectra the line branching analysis could be successfully used for evaluation of Ar 1s state densities, based on the pairs of the emission lines originating from different $\operatorname{Ar}(2 \mathrm{p})$ excited states. Evaluated $\operatorname{Ar}(1 \mathrm{~s})$ states densities are further used as an input parameter in the rate balance equations describing populations of $\operatorname{Ar}(2 \mathrm{p})$ states.

Steady state solutions of the rate balance equations, for different $\operatorname{Ar}(2 p)$ levels, were 
further used to describe emitted radiation including the radiation trapping effects (self-

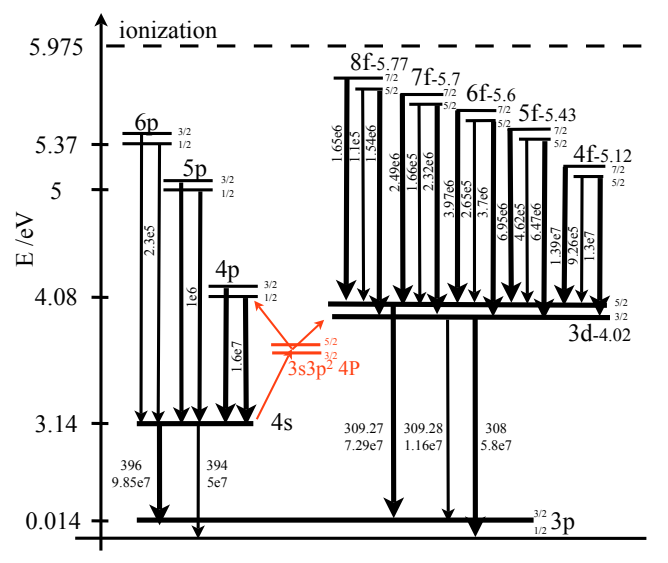

FIG. 6. (Colour online) Energy levels of $\mathrm{Al}$ atoms and radiative transitions.

From Figs. 5 and 6 one can see that the observed Al spectral lines are composed of two sets of transitions originating from the lower excited $\mathrm{Al}(4 \mathrm{~s})$ and $\mathrm{Al}(3 \mathrm{~d})$ states. Emission lines at 394 and $396 \mathrm{~nm}$ are clean single transitions originating from a single excited $\mathrm{Al}(4 \mathrm{~s})$ state. The second set of lines is actually composed of three lines: one line at $309.27 \mathrm{~nm}$ originating from the energetically higher multiplet $\mathrm{Al}(3 \mathrm{~d}[5 / 2])$, a second line at 309.28 line originating from the lower multiplet $\mathrm{Al}(3 \mathrm{~d}[3 / 2])$, and third line of this set is a pure single transition emitted from the lower $\mathrm{Al}(3 \mathrm{~d}[3 / 2])$ multiplet. The first two lines make a double line which cannot be resolved with the spectrometer used in the experiment. 
In the case of aluminium, the rate balance equations describing the population of excited $4 \mathrm{~s}$ and $3 \mathrm{~d}$ states includes the electron impact excitation from ground states and the radiative decay from higher levels. However, in contrast to Ar plasmas where Ar atom density was known, the aluminium atom density in the plasma is an unknown variable used for the evaluation of the sputtering-deposition processes. The aluminium density is estimated by including $\mathrm{Al}$ line branching equations into the $\mathrm{Ar} / \mathrm{Al} \mathrm{CRM}$.

\section{Results}

Measured optical emission spectra were first analysed using only Ar emission lines, ignoring possible interactions between $\mathrm{Ar}$ and $\mathrm{Al}$. Ar spectra were first analysed by the line branching method considering three pairs of lines originating from different $\operatorname{Ar}(2 \mathrm{p})$ levels in order to extract the densities of $\operatorname{Ar}(1 \mathrm{~s} 5,1 \mathrm{~s} 4)$ excited states. For this purpose the following ratios of line pairs were considered: 727/696, 738/706 and 800/763 originating from $\operatorname{Ar}(2 \mathrm{p} 2)$, $\operatorname{Ar}(2 \mathrm{p} 3)$ and $\operatorname{Ar}(2 \mathrm{p} 6)$ excited levels, respectively.

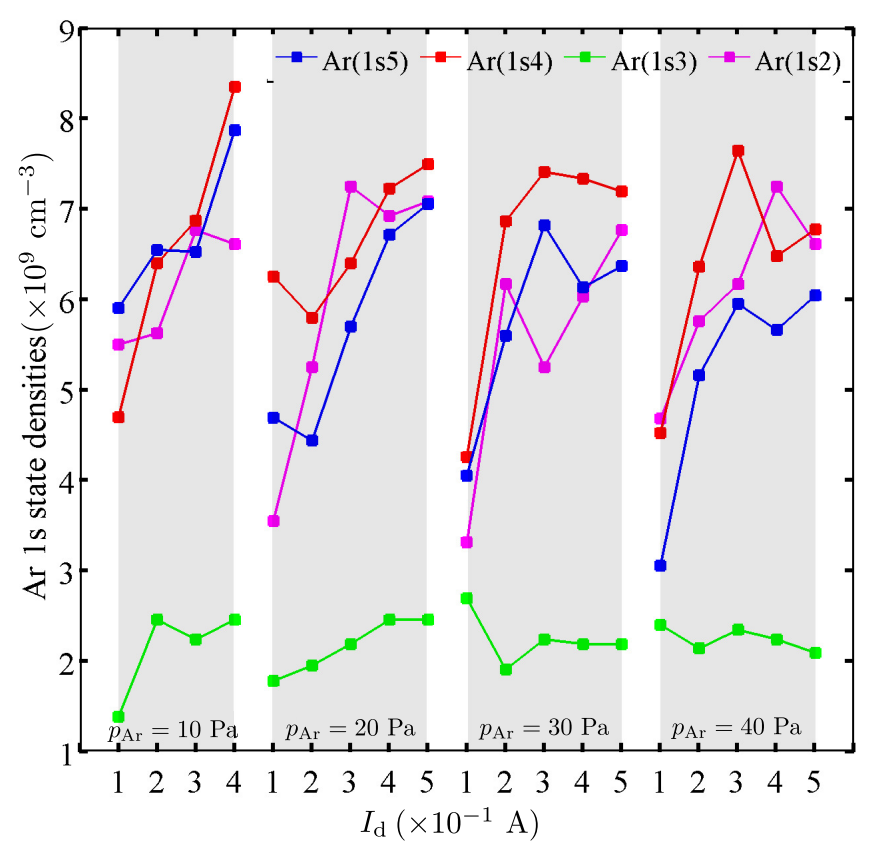

FIG. 7. (Colour online) Evaluated densities of Ar 1s states.

Once these two values were known similar line ratios could be recovered to evaluate the densities of other $\operatorname{Ar}(1 \mathrm{~s} 3)$ and $\operatorname{Ar}(1 \mathrm{~s} 2)$ excited states. Due to the limited observed spectral 
range, single line ratios were constructed for the evaluation of each of the two states: the 826/696 line ratio for the $\operatorname{Ar}(1 \mathrm{~s} 2)$ state and the $794 / 714$ line ratio for the $\operatorname{Ar}(1 \mathrm{~s} 3)$ state. Evaluations of $\operatorname{Ar}(1 \mathrm{~s} 2)$ and $\operatorname{Ar}(1 \mathrm{~s} 3)$ states densities were prone to the error due to low intensity of the $714 \mathrm{~nm}$ line and the reduced spectral sensitivity of the spectrometer (the $826 \mathrm{~nm}$ line is at the edge of the observable spectral range). The reconstructed densities of all argon 1s states for the considered plasma conditions are shown in Fig. 7.

With known $\operatorname{Ar}(1 \mathrm{~s} 4,1 \mathrm{~s} 5)$ state densities, rate balance equations (Eq. (2)) were constructed for the excitation of $6 \operatorname{Ar}(2 \mathrm{p})$ levels $\left(2 \mathrm{p}_{1}, 2 \mathrm{p}_{3}, 2 \mathrm{p}_{5}, 2 \mathrm{p}_{6}, 2 \mathrm{p}_{7}, 2 \mathrm{p}_{8}, 2 \mathrm{p}_{9}\right)$ resulting in a steady state solution depending only on electron temperature. Line ratios were then simulated for a wide range of electron temperatures and compared with the measured values in order to find the best match. Such a method was developed and discussed in details in Ref. [46] and compared with a complete CRM [44] for similar plasma conditions. Rate coefficients for electron impact excitation were calculated using the same set of excitation cross-sections as in Refs. [43, 44] and downloaded from the LXCAT database [52] and integrated over the desired electron energy distribution function. Rate coefficients calculated for Maxwell electron energy distribution function showed best agreements for most of line ratios. The resulting estimates of the average electron temperature based on argon CRM are presented in Fig. 8 in thick blue with solutions from individual line ratios shown in the background with thin green, cyan, magenta and black lines. Only the results with satisfactory confidence were shown in the figure, based on the analysis of a wide range of conditions discussed in Ref. [46]. Evaluated electron temperatures are relatively low $(\sim 1.5 \mathrm{eV})$ and show systematic dependence on pressure and power.

The electron temperature for each studied discharge condition was then used to reconstruct and compare the relative changes in the intensities of the lines and estimate the relative changes in the electron densities considering that the measurements conditions remained identical between each experiment. The estimated relative changes in electron densities are presented in the Fig. 9 (blue lines).

Analysis of the $\mathrm{Al}$ spectra was done by building a simpler CRM due to the limited number of available cross-sections and known interactions responsible for the population of $\mathrm{Al}$ levels. A set of cross-sections for electron impact excitation from ground state, available for only a limited number of transitions, is taken from Ref. [53] and is used for calculations of rate coefficients. Einstein coefficients for spontaneous emission of Al levels were taken from the 


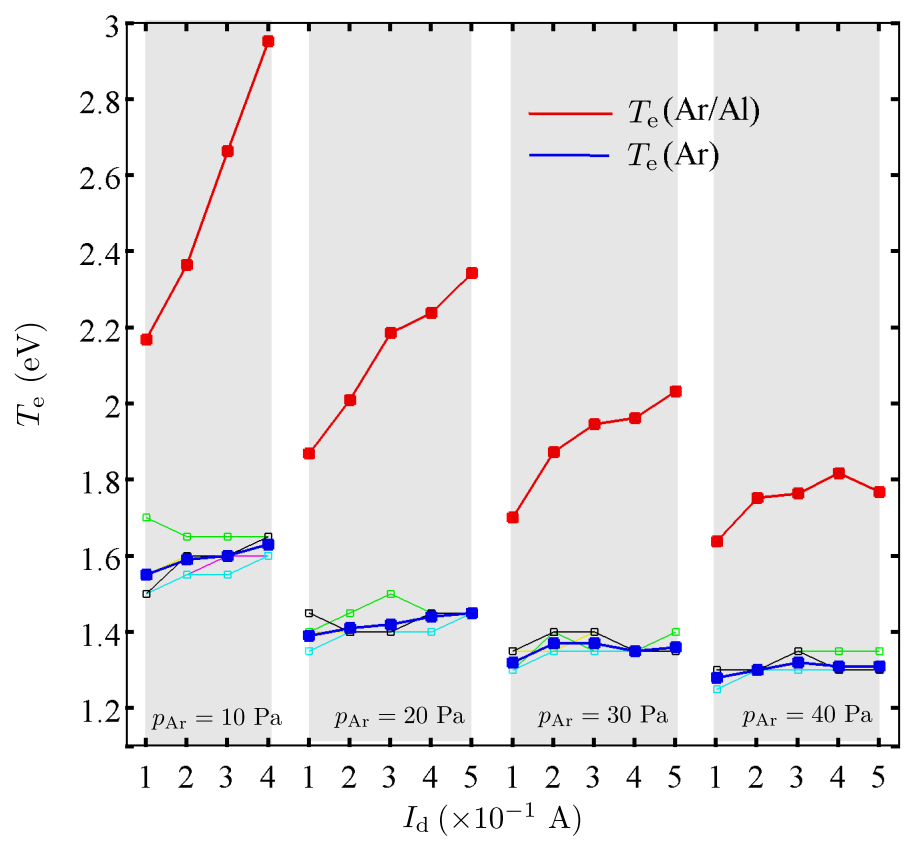

FIG. 8. (Colour online) Electron temperature estimation based on pure argon CRM - Te(Ar) and $\mathrm{Ar} / \mathrm{Al}$ line ratio - $\mathrm{Te}(\mathrm{Ar} / \mathrm{Al})$. The estimates of the average electron temperature based on argon CRM is the thick blue line. Solutions from individual line ratios are shown in the background with thin green, cyan, magenta and black lines. The hybrid Ar/Al model solution is the thick red line.

NIST database [51].

The population of the energetic levels shown in Fig. 6 are modelled by taking into account direct electron impact excitation from ground state (from both multiplets) while for lower energetic states $\mathrm{Al}(4 \mathrm{~s})$ and $\mathrm{Al}(3 \mathrm{~d})$, additional population by cascading radiation from upper levels was accounted for. In this description, no difference between the multiplets was considered in the collisional processes so that excitation from total ground state density was taken in calculations. Considering that the CRM based on the line ratio method is only dependent on the electron temperature, the description of $\mathrm{Al}$ levels will additionally have two more unknowns which are the ground state density multiplets. Observed emission spectra of $\mathrm{Al}$ consists of only a few strong emission lines originating from three different energetic levels. It limits the construction of a closed system of equations which would provide a unique solution. For this purpose, the Ar $750 \mathrm{~nm}$ line was also included in the description and used for comparison with Ar emission lines.

For this purpose, the ratio of $\mathrm{Ar} 750 \mathrm{~nm}$ and $\mathrm{Al} 308 \mathrm{~nm}$ lines was used to construct one 


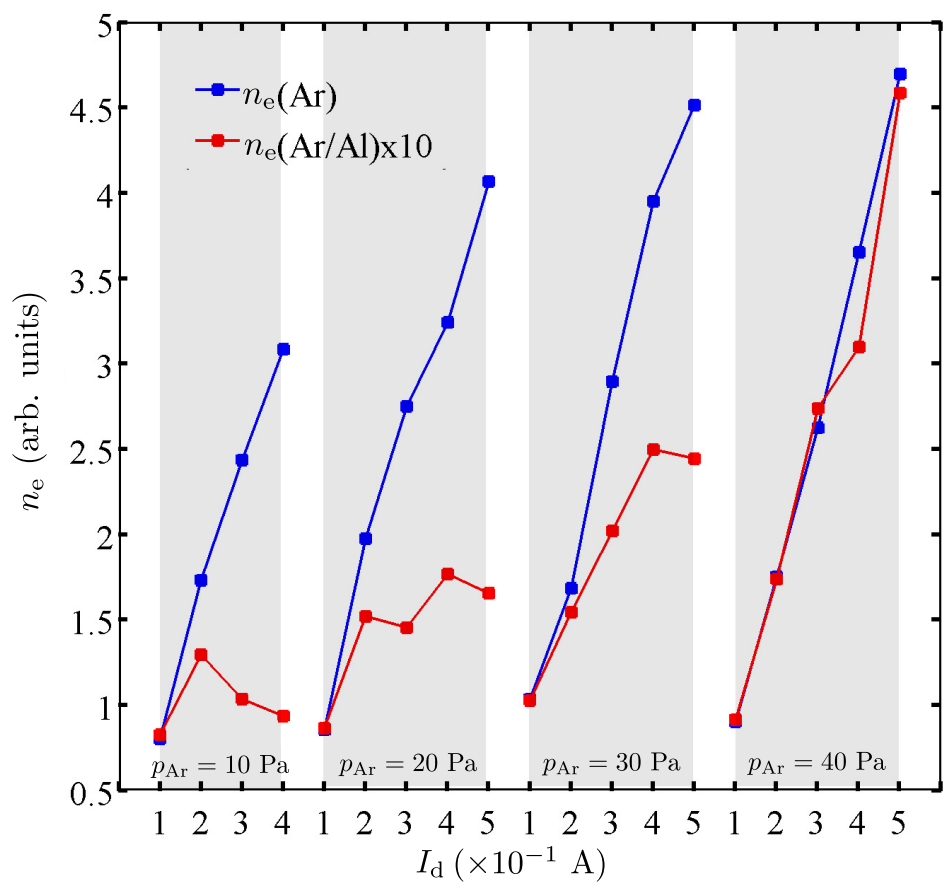

FIG. 9. Relative changes in the electron densities evaluated for the electron temperatures based on pure Argon ( $\mathrm{Te}(\mathrm{Ar})$ in blue) and Argon/Aluminium ( $\mathrm{Te}(\mathrm{Ar} / \mathrm{Al})$ in red) analysis.

equation while the other was built by comparing the intensities of $\mathrm{Al} 308$ and $\mathrm{Al} 309 \mathrm{~nm}$ aluminium spectral lines. A third equation accounting for the radiation from the excited $\mathrm{Al}(4 \mathrm{~s})$ state was also considered in the model as a ratio of the $\mathrm{Al} 394 \mathrm{~nm}$ and $\mathrm{Ar} 750 \mathrm{~nm}$ lines. The system of equations was additionally extended and improved by an additional line branching equation correlating the ratio of Al 394/396 nm lines with the ground state multiplet densities. Therefore, the line branching equation was constructed to evaluate the ratio of 394/396 $\mathrm{nm}$ emission lines originating from the same $\mathrm{Al}(4 \mathrm{~s})$ exited level decaying on different ground state multiplets. With one more set of lines with the same properties (originating from the same level and decaying to the same ground multiplets) it would be possible to uniquely solve the system and determine the $\mathrm{Al}$ atom density in the plasma without additional modelling. However, in the presented measurements, a set of such lines was not observed. The best candidate would be the ratio of 265.24/266.03 nm lines originating from $\mathrm{Al}(5 \mathrm{~s})$ excited state which is in a lower UV range usually not observable with standard (VIS-NIR) optics. However, the ratio of the Einstein coefficients of Al $394 \mathrm{~nm}$ and $396 \mathrm{~nm}$ lines is close to 0.5 while the measured ratio is close to unity, see Fig. 5. This difference can be used to describe the necessary density ratio of the ground state multiplets based on 


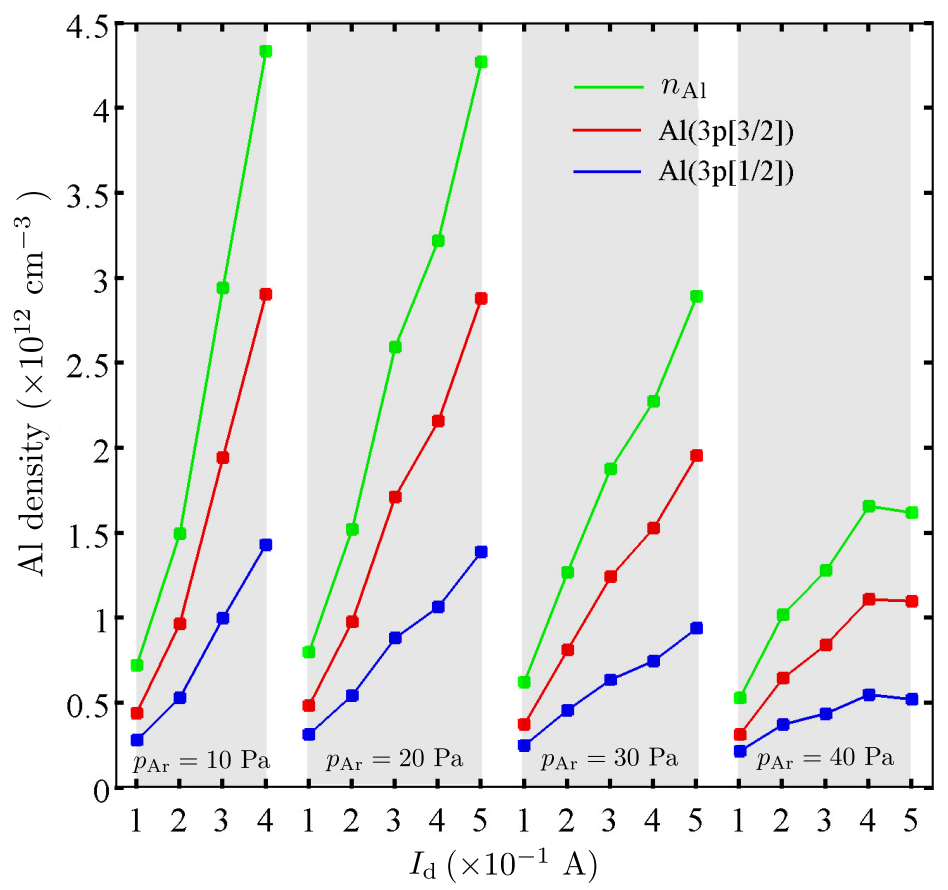

FIG. 10. Estimated number densities of Aluminium ground state multiplets.

self-absorption.

Hybrid $\mathrm{Ar} / \mathrm{Al}$ model extended with the line branching relation consists of enough equations for finding a unique solution to the electron temperature and the density of aluminium in the ground state in the plasma. The evaluated electron temperature is presented in Fig. 8 (thick red line). Although the temperature shows similar systematic changes with controlled parameters, the absolute values are substantially higher that the one based on pure argon analysis. Based on this electron temperatures, reconstructed relative changes in electron densities appear lower, see Fig. 9. The density of $\mathrm{Al}$ in the plasma, evaluated by hybrid $\mathrm{Ar} / \mathrm{Al}$ analysis, is presented in Fig. 10 for each multiplet. The aluminium atom density is evaluated to be on the order of $10^{12} \mathrm{~cm}^{-3}$, directly increasing with increasing plasma current and decreasing with pressure.

\section{DISCUSSION}

Results obtained by different methods and techniques should be carefully analysed accounting for strong spatial inhomogeneity of the DC magnetron discharge and redistribution of the plasma glow due to changes of pressure and power. Therefore the measurements fur- 
ther away from the cathode (electric probe) provide values characteristic of positive columns of DC magnetron discharges, while measurements closer to the cathode (OES) characterise the more energetic regions of the DC magnetron discharge (the high-density plasma ring confined by the magnetic arch). Nevertheless, both spectroscopy and Langmuir probe measurements show common trends of plasma parameters as a function of the working pressure, $p_{\text {Ar }}$, and the current discharge, $I_{\mathrm{d}}$, but also present a few differences:

- At fixed $I_{\mathrm{d}}$, an increase of the background gas pressure $p_{\text {Ar }}$ results in a decrease of the electron temperature. The trend for the electron density is not so clear: Langmuir probe measurements indicate a small decrease in the electron density when increasing the pressure while spectroscopy measurements using argon lines tend to show a slight increase up to $30 \mathrm{~Pa}$. Above $30 \mathrm{~Pa}$, it depends on $I_{\mathrm{d}}$. The hybrid Ar/Al CRM seem to favour a constant increase of electron density.

Langmuir probe measurement being preformed relatively far away from the "active" plasma ring gave much lower values of $T_{\mathrm{e}}$ than spectroscopic measurements $\left(T_{\mathrm{e}} \sim\right.$ $0.60 \pm 0.05 \mathrm{eV}$ compared to $T_{\mathrm{e}} \sim 1.5 \pm 0.2 \mathrm{eV}$ for the Ar CRM). Similar experiments performed with a tungsten cathode have shown that $T_{\mathrm{e}}$ is decreasing on the axis of the discharge when moving away from the cathode [33], explaining the difference in the reported values. However, the reported electron temperature is of the same order as the one found at the same position in the discharge when using a tungsten cathode $\left(T_{\mathrm{e}} \sim 0.6 \mathrm{eV}\right.$ for $\mathrm{Al}$ and $T_{\mathrm{e}} \sim 1 \mathrm{eV}$ for $\mathrm{W}$ for $I_{\mathrm{d}}=300 \mathrm{~mA}$ and $\left.p_{\mathrm{Ar}}=30 \mathrm{~Pa}[33]\right)$, and very close to the electron temperature reported for experiments in the plasma of the condensation chamber of a MS-GAS ( $T_{\mathrm{e}} \simeq 0.5 \mathrm{eV}$ for a titanium cathode, $I_{\mathrm{d}}=300 \mathrm{~mA}$ and $\left.p_{\mathrm{Ar}}=34.5 \mathrm{~Pa}[24]\right)$. The measured plasma densities were in agreement with those reported in the literature $[24,33]$ and with only a slight dependence to the gas pressure.

Spectroscopic measurements made just below the cathode and going through the high density plasma ring unsurprisingly revealed a higher $T_{\mathrm{e}}$ but exhibited the same trend as the Langmuir probe measurements. Using Ar CRM, $T_{e}$ decreased from $T_{\mathrm{e}} \simeq 1.6 \mathrm{eV}$ at $p_{\mathrm{Ar}}=10 \mathrm{~Pa}$ to $T_{\mathrm{e}} \simeq 1.3 \mathrm{eV}$ at $p_{\mathrm{Ar}}=40 \mathrm{~Pa}$ and showed little sensitivity to the intensity of the discharge current (i.e. a small increase of $T_{\mathrm{e}}$ is observed). This range of $T_{\mathrm{e}}$ is in agreement with the value reported in the literature for the electron temperature in the plasma ring of the condensation chamber of a MS-GAS [27]. The simultaneous 
use of aluminium and argon lines (hybrid A/Ar CRM) gave higher $T_{\mathrm{e}}$. However the ture. The main difference is that now the electron temperature reacts strongly to the discharge current, especially at the lowest investigated pressure. Both spectroscopic models have their drawbacks that could explain observed discrepancies in electron temperature. Due to the low density of $\operatorname{Ar}(1 \mathrm{~s})$ states, the excitation from ground state Ar by energetic electrons is the dominant process and secondary excitation are negligible making the whole method impervious to low energetic electrons $[46,49]$ and resulting in large uncertainties in electron temperature. The evaluated electron temperatures were used for an estimation of relative changes of electron density resulting in a different overall response of the system.

- At fixed background gas pressure $p_{\mathrm{Ar}}$, an increase of the current results, as expected, in an increase of the plasma density and the electron temperature is marginally affected. Langmuir probe measurements on the axis of the discharge assembly and far below the plasma ring confined below the cathode showed density on the order of $n_{\mathrm{e}} \sim 10^{10} \mathrm{~cm}^{-3}$, which is comparable to what can be found in the literature [24, 33]. The plasma density in the dense plasma ring below the cathode is one to two orders of magnitude higher [24, 33].

Spectroscopy studies showed a linear increase of the relative plasma density with current when using the Ar CRM. The hybrid Ar/Al CRM did not show a linear increase of $n_{\mathrm{e}}$ at low pressure. The differences in $T_{\mathrm{e}}$ and $n_{\mathrm{e}}$ obtained from the Ar CRM and the hybrid $\mathrm{Al} / \mathrm{Ar} \mathrm{CRM}$ can be explained by the fact that low energetic electrons are directly included in the hybrid $\mathrm{Ar} / \mathrm{Al} \mathrm{CRM}$ since the excitation of $\mathrm{Al}$ states is possible at electron energies as low as $\sim 3 \mathrm{eV}$. However the modelling of the excitation of $\mathrm{Al}$ states requires a good knowledge of $\mathrm{Al}$ collisional-radiative coupling constant and of the cross-sections of excitation processes. Due to limited data on such processes only a basic corona model was used for the modelling of $\mathrm{Al}$ excited state. Thus, in case of pure Ar CRM, changes of the discharge current is mostly reflected in electron density variations while in the hybrid analysis $T_{\mathrm{e}}$ is more sensitive to the current change. This discrepancy is most pronounced on lower pressure and slowly vanishes with pressure increase. 
- In addition to $T_{\mathrm{e}}$ and $n_{\mathrm{e}}$, spectroscopic studies provided the evolution of the densities $1 \mathrm{~s}_{3}$ state is revealed to be almost insensitive to both background gas pressure and discharge current even though it seems that at low pressure $(10 \mathrm{~Pa}$ and $20 \mathrm{~Pa})$ an increase of the current results in an increase of the $1 \mathrm{~s}_{3}$ density. All other states show an increase of their densities with increasing current and tends to exhibit a slight decrease in density when increasing pressure at fixed current. This is particularly visible for the $1 \mathrm{~s}_{5}$ metastable state. Overall $\operatorname{Ar}(1 \mathrm{~s})$ state densities are not very high compared with neutral Ar density making excitation from ground state the dominant process. As previously stated, under such conditions the evaluation of electron temperature based on the line ratio is less accurate and predominantly accounts for high energetic electrons in the electron energy distribution function, as discussed in [46]. As a result evaluated electron temperature can be systematically over/under estimated due to the small errors in measurements, calibration procedure or data processing.

The evaluation of $T_{\mathrm{e}}$ by the hybrid model is directly coupled to the aluminium atom density through excitation processes and self-absorption of $\mathrm{Al}$ emission lines which is quite strong in our experimental conditions. The resulting aluminium atom densities were thus estimated to be in the range $10^{11}-10^{12} \mathrm{~cm}^{-3}$. This can be additionally justified considering that the line branching ratio of $\mathrm{Al}(394) / \mathrm{Al}(396)$ lines imposes a lower limit of $\mathrm{Al}$ atom density of about $5 \times 10^{16} \mathrm{~m}^{-3}$. Al densities reported in this work are slightly higher than the ones reported in [54] which was operated on drastically lower pressures and close to the values reported for the sputtering of aluminium or titanium under similar conditions $[55,56]$. Note that the evaluation of $\mathrm{Al}$ densities could be improved and simplified by including one more pair of Al lines (preferably $\mathrm{Al}(265) / \mathrm{Al}(266))$ originating in deeper UV range.

In order to understand the influence of discharge conditions and the presence of aluminium atoms on plasma parameters, a simple global particle and power balance model for steady state discharge is used. The model follows the methodology found in Refs. [41, 54]. The plasma is reduced to a homogeneous half torus of mean radius $\bar{R}$ and of radius $R_{\text {tor }}$ confined above the cathode racetrack [54]. In our experiment $\bar{R} \simeq 2.2 \mathrm{~cm}$ is the mean radius of the racetrack and $R_{\text {tor }} \simeq 0.5 \mathrm{~cm}$ is the half-width of the racetrack (see Fig. 1). The plasma volume is therefore $V_{\text {plasma }}=\pi^{2} \bar{R} R_{\text {tor }}^{2}$ and the bounding surface 
area $A_{\text {plasma }}=A_{\text {planar }}+A_{\text {tor }}$ is comprised of the planar contribution above the racetrack $A_{\text {planar }}=\pi\left[\left(\bar{R}+R_{\text {tor }}\right)^{2}-\left(\bar{R}-R_{\text {tor }}\right)^{2}\right]$ and the curved contribution $A_{\text {tor }}=2 \pi \bar{R} R_{\text {tor }}$. The effect of the background gas pressure, the amplitude of the cathode voltage and the discharge current on the width and height of the "plasma ring" [38, 39, 57] are ignored. However, the fraction of ions $f_{t}$ leaving the plasma towards the target is left as a free parameter in order to adjust the discharge current, voltage and power, $P=I_{\mathrm{d}} V_{c}$ with the experimental ones. The model includes only the electron density $n_{\mathrm{e}}$, the background neutral argon density $n_{\mathrm{Ar}}$ and the thermalised aluminium atom density $n_{\mathrm{Al}}$. Direct impact ionisation of argon and aluminium as well as Penning ionisation of aluminium through collisions with argon metastable atoms are considered. It was indeed shown that ionisation of sputtered metal due to Penning ionisation through impact with metastable states of the argon is important in magnetron discharges and consistent with diffusive losses of argon meta- stables [58, 59]. The particle balance equation, the power balance equation and the experimental adjustment of $f_{t}$ equations are:

$$
\begin{aligned}
A_{\mathrm{planar}} h_{\ell} u_{\mathrm{B}} n_{\mathrm{e}}= & n_{e} V_{\mathrm{plasma}} f_{t} \cdot\left[n_{\mathrm{Ar}} K_{\mathrm{iz}, \mathrm{Ar}}\right. \\
& +n_{\mathrm{Al}} K_{\mathrm{iz}, \mathrm{Al}} \\
& \left.+n_{\mathrm{Ar}} K_{\mathrm{ex}, \mathrm{Ar}(4 \mathrm{~s})} \tau_{A r^{*}} n_{\mathrm{Al}} \sigma_{p} v_{T_{\mathrm{Al}}}\right], \\
I_{\mathrm{d}} V_{c}= & P_{\text {loss }, \mathrm{e}}+P_{\text {loss }, \mathrm{w}}, \\
\frac{I_{\mathrm{d}}}{e(1+\gamma)}= & A_{\text {planar }} h_{\ell} u_{\mathrm{B}} n_{\mathrm{e}},
\end{aligned}
$$

where $h_{\ell}=0.80 / \sqrt{4+R_{\text {tor }} / \lambda_{\text {in }}}$ is the effective area correction factor due to the variation of plasma density from the middle to the edge [41] in a plasma regime where transport is diffusive and ambipolar [60], $\lambda_{i n}=1 /\left(n_{\mathrm{Ar}} \sigma_{i n}\right)$ is the ion-neutral mean free path where $\sigma_{i n}=10^{-18} \mathrm{~m}^{2}[41], u_{\mathrm{B}}=\sqrt{k_{\mathrm{B}} T_{\mathrm{e}} / m_{\mathrm{e}}} \cdot\left(1+\pi \lambda_{D e} /\left(2 \lambda_{i n}\right)\right)^{-0.5}$ is the ion-collision corrected Bohm velocity [41] where $\lambda_{D e}$ is the electron Debye length at the cathode sheath edge, $K_{\mathrm{iz}, \mathrm{Ar}}$ is the argon electron impact direct ionisation rate, $K_{\mathrm{iz}, \mathrm{Al}}$ is the aluminium atom electron impact direct ionisation rate, $K_{\text {ex, } \operatorname{Ar}(4 \mathrm{~s})}$ is the argon excitation rate towards metastable $4 \mathrm{~s}$ levels, $\tau_{A r^{*}}$ is the lifetime of argon metastable, $\sigma_{p}=7.15 \cdot 10^{-19} \mathrm{~m}^{2}$ is the Penning ionisation cross-section of aluminium [58], $v_{T_{\mathrm{A} 1}}$ is the thermal velocity of aluminium atoms, and $\gamma=0.1$ the effective ion-induced secondary electron emission coefficient which here is considered here. The argon metastable lifetime is given by $\tau_{A r^{*}}^{-1}=\tau_{D}^{-1}+\tau_{\mathrm{m}-\mathrm{e}}^{-1}$ where the diffusion lifetime 
is $\tau_{D}^{-1}=K_{2} n_{\mathrm{Ar}}+K_{3} n_{\mathrm{Ar}}^{2}+\frac{D_{0}}{n_{\mathrm{Ar}}} \Lambda^{-2}$ with $K_{2}=2.3 \cdot 10^{-15} \mathrm{~cm}^{3} \mathrm{~s}^{-1}, K_{3}=1.4 \cdot 10^{-36} \mathrm{~cm}^{6} \mathrm{~s}^{-1}$, and $D_{0}=2.4 \cdot 10^{18} \mathrm{~cm}^{-1} \mathrm{~s}^{-1}[61]$, and the electron-metastable collision frequency is $\tau_{\mathrm{m}-\mathrm{e}}^{-1}=$ $5 \cdot 10^{-8} n_{\mathrm{e}} T_{\mathrm{e}}$ with $n_{\mathrm{e}}$ in $\mathrm{cm}^{-3}$ and $T_{\mathrm{e}}$ in $\mathrm{eV}[58]$.

The power loss to the walls $P_{\text {loss,w }}$ carried by the particles lost at the plasma bounding surface is given by $[41,54]$ :

$$
\begin{aligned}
P_{\text {loss }, \mathrm{w}}=\frac{I_{\mathrm{d}}}{e(1+\gamma) f_{t}}( & \frac{5}{2} k_{\mathrm{B}} T_{\mathrm{e}}+0.75 f_{t} e\left|V_{c}\right| \\
& \left.+\left(1-f_{t}\right) k_{\mathrm{B}} T_{\mathrm{e}} \ln \left(\sqrt{\frac{m_{\mathrm{Ar}}}{2 \pi m_{\mathrm{e}}}}\right)\right) .
\end{aligned}
$$

The collisional power loss $P_{\text {loss,e }}$ are given by [41, 54]:

$$
P_{\text {loss }, \mathrm{e}}=n_{\mathrm{e}} V_{\text {plasma }}\left[n_{\mathrm{Ar}} K_{\mathrm{iz}, \mathrm{Ar}} \epsilon_{\mathrm{c}, \mathrm{Ar}}+n_{\mathrm{Ar}} K_{\mathrm{iz}, \mathrm{Al}} \epsilon_{\mathrm{c}, \mathrm{Al}}\right],
$$

where the effective energy required per created electron-ion pair is given by $[41,54]$ :

$$
\epsilon_{\mathrm{c}, \mathrm{s}}=\left[\epsilon_{i z, s}+\sum_{j} \frac{K_{\mathrm{ex}, \mathrm{s}_{\mathrm{j}}}}{K_{\mathrm{iz}, \mathrm{s}}} \epsilon_{\mathrm{ex}, \mathrm{s}_{\mathrm{j}}}+\frac{K_{\mathrm{el}, \mathrm{s}}}{K_{\mathrm{iz}, \mathrm{s}}} \frac{3 m_{\mathrm{e}}}{m_{\mathrm{s}}} k_{\mathrm{B}} T_{\mathrm{e}}\right] .
$$

Species $s \in[\mathrm{Ar}, \mathrm{Al}]$ of atomic mass $m_{s}$ interact with electron of mass $m_{\mathrm{e}} \cdot \epsilon_{i z, s}$ and $\epsilon_{\mathrm{ex}, \mathrm{s}_{\mathrm{j}}}$ are the respective ionisation energies and excitation energies of the $\mathrm{j}^{\text {th }}$ level with the corresponding reaction rate coefficient $K_{\mathrm{iz}, \mathrm{s}}$ and $K_{\mathrm{ex}, \mathrm{s}_{\mathrm{j}}} . K_{\mathrm{el}, \mathrm{s}}$ is the elastic collision rate coefficients. In Fig. 11, the reaction rate coefficients are calculated for for argon and aluminium were calculated assuming a Maxwellian electron energy distribution function (EEDF) and using the cross sections from Refs. [53,62]. The $4 \mathrm{~s}, 4 \mathrm{p}$ and $4 \mathrm{~d}$ levels of argon are considered and the excited levels of aluminium are grouped under one rate constant with an excitation energy $\epsilon_{\mathrm{ex}, \mathrm{Al}}=3.13 \mathrm{eV}$. As can be seen, for electron temperatures around 1-2 eV, the reaction rate constants of aluminium are much higher than the ones for argon and one can expect moderate quantities of aluminium atoms in the plasma to have an impact on plasma parameters. Eqs. (3-5) were solved numerically for different aluminium atom densities over the experimental range of $I_{\mathrm{d}}$ and $p_{\mathrm{Ar}}$. The cathode voltage $V_{c}$ is taken as the experimental one measured for a given set of $I_{\mathrm{d}}$ and $p_{\mathrm{Ar}}$ (see Fig. 2). The temperature of the background $\mathrm{Ar}$ gas and thermalised aluminium atoms is set at $T=400 \mathrm{~K}$ which is close to the expected temperature [56]. The results of the calculation are shown in Fig. 12.

As expected, $T_{\mathrm{e}}$ decreases whith increasing pressure. The calculated values are not far from the ones recovered using spectroscopic measurements but due to the numerous approximations of the model only qualitative comparison can be done. One can also see that 


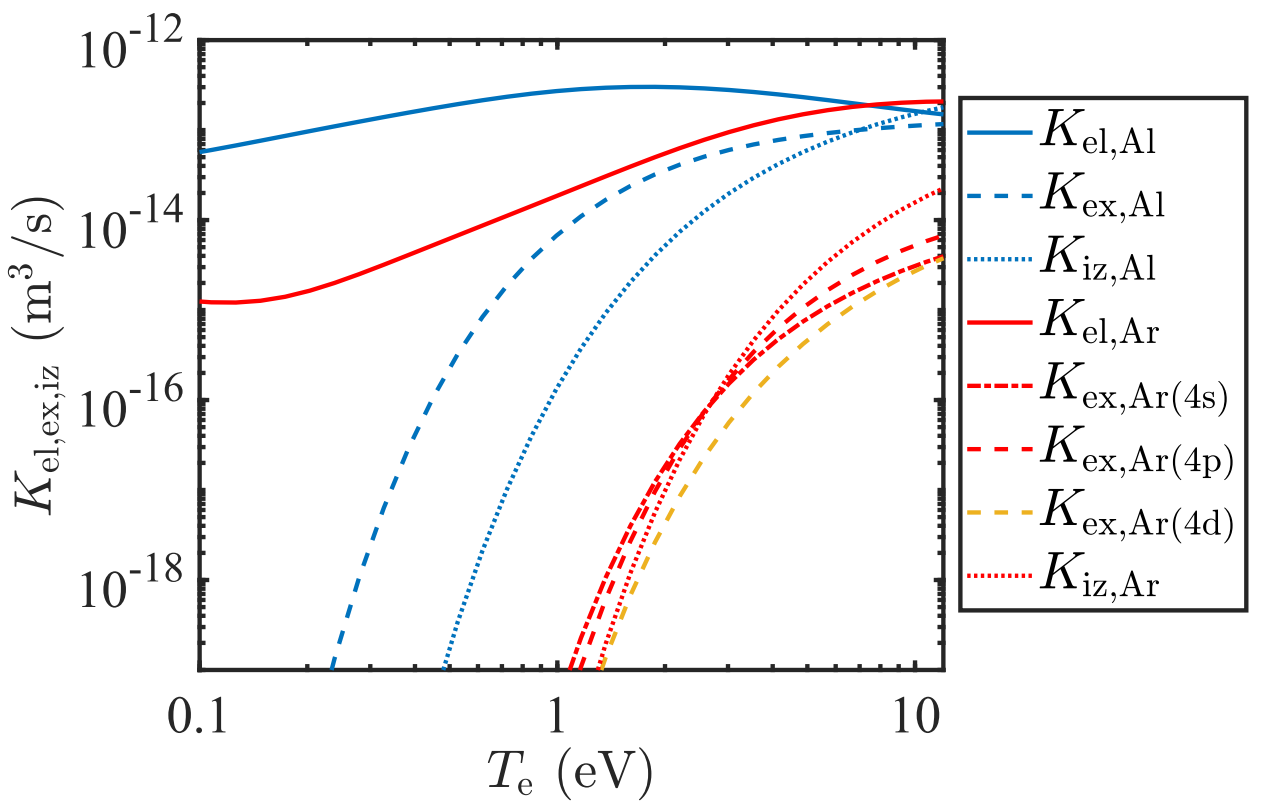

FIG. 11. (Colour online) Reaction rate constants as a function of $T_{\mathrm{e}}$ for argon (red curves) and aluminium (blue curves). The reaction rate for argon and aluminium were calculated assuming a Maxwellian EEDF and using the cross sections from Refs. [53, 62]

the presence of a small density of aluminium atoms $\left(n_{\mathrm{Al}} \simeq 5 \cdot 10^{12} \mathrm{~cm}^{-3}\right.$ corresponding to less than $0.3 \%$ of $n_{\mathrm{Ar}}$ at $p_{\mathrm{Ar}}=10 \mathrm{~Pa}$ ) is enough to decrease the electron temperature by a few percent and might partially explain the relatively low measured electron temperatures. Increasing the discharge current results in a small increase in the electron temperature and a linear increase in the plasma density. Note that the plasma density is almost unaffected by the presence of aluminium. The fraction of ions $f_{t}$ leaving the plasma towards the target (not shown here) changed from around $f_{t} \sim 0.6$ at $p_{\mathrm{Ar}}=10 \mathrm{~Pa}$ to $f_{t} \sim 0.9$ at $p_{\mathrm{Ar}}=40 \mathrm{~Pa}$. However, our simple model cannot be used to obtained quantitative values since many relevant physical phenomena are not included. For instance, increasing pressure leads to lower sputtering rate due to lower energy of the ion hitting the cathode. Moreover, the influence of power and pressure on the width of the racetrack is not taken into account, nor is the thermalisation of sputtered atom and the heating of the background gas. Therefore, a high discharge current might not necessarily lead to more sputtering and a higher aluminium atom density in the plasma. For example, at the highest background gas pressure, collisions can result in a lower mean energy of the ion hitting the cathode and therefore a smaller sputtering rate and a reduced aluminium density (see Fig. 10) 

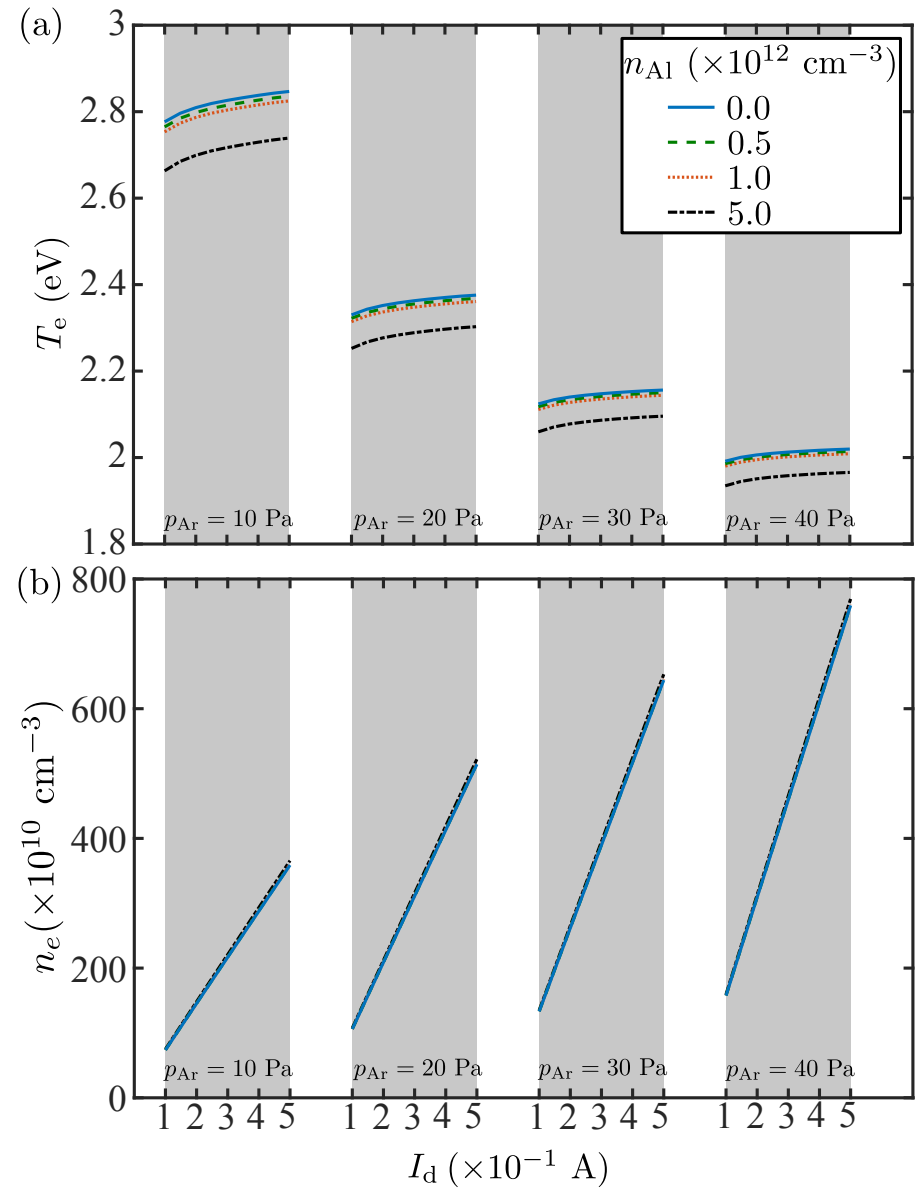

FIG. 12. (Colour online) Calculated plasma parameters as a function of $p_{\text {Ar }}$ and $I_{d}$. (a) Electron temperature, $T_{\mathrm{e}}$. (b) Electron density, $n_{\mathrm{e}}$.

\section{CONCLUSION}

In this article, the plasma parameters of an aluminium cathode direct-current magnetron discharge in argon with operating pressures ranging from $10 \mathrm{~Pa}$ to $40 \mathrm{~Pa}$ and discharge currents ranging from $100 \mathrm{~mA}$ to $500 \mathrm{~mA}$ were explored. The density of argon excited states, the sputtered aluminium atom density and the electron temperature were estimated using a collisional-radiative model and compared to plasma parameters obtained from Langmuir probe measurements. The trends of electron temperature and plasma density obtained using optical emission spectroscopic data agrees well with probe measurements. The differences in reported values are explained by the different locations in the discharge that were studied using spectroscopy and Langmuir probes. However, the reported electron temperature were lower than the values found in the literature for similar discharge conditions and different 
cathode material [33]. By using a simple global model, the influence of the discharge parameters, namely the background argon pressure and the discharge current, as well as the density of sputtered aluminium atoms was investigated. It was shown that the presence of aluminium atoms in the plasma cools down the electrons and but has little effect on the plasma density. This is most probably due to the lower excitation and ionisation thresholds of aluminium with respect to argon.

Future studies will require the development of a more complete hybrid CRM. It will allow more precise derivation of plasma parameters. Direct measurement of the aluminium atom density through absorption spectroscopy will also be performed. A careful modelling of the magnetron discharge should also be done in order to study the influence of sputtering and transport of aluminium atoms on plasma parameters.

\section{ACKNOWLEDGMENTS}

LC acknowledges the support of the Natural Sciences and Engineering Research Council of Canada (NSERC), RGPIN-2019-04333.

[1] W. D. Gill and E. Kay, Efficient low pressure sputtering in a large inverted magnetron suitable for film synthesis, Review of Scientific Instruments 36, 277 (1965).

[2] R. K. Waits, Planar magnetron sputtering, Journal of Vacuum Science and Technology 15, 179 (1978).

[3] T. E. Sheridan, M. J. Goeckner, and J. Goree, Model of energetic electron transport in magnetron discharges, Journal of Vacuum Science \& Technology A: Vacuum, Surfaces, and Films 8, $30(1990)$.

[4] D. Depla and W. Leroy, Magnetron sputter deposition as visualized by monte carlo modeling, Thin Solid Films 520, 6337 (2012).

[5] M. L. Escrivão, A. M. C. Moutinho, and M. J. P. Maneira, Planar magnetron glow discharge on copper: Empirical and semiempirical relations, Journal of Vacuum Science \& Technology A 12, 723 (1994).

[6] S. M. Rossnagel and J. Hopwood, Metal ion deposition from ionized mangetron sputtering 
discharge, Journal of Vacuum Science \& Technology B: Microelectronics and Nanometer Structures Processing, Measurement, and Phenomena 12, 449 (1994).

[7] K. Matyash, M. Fröhlich, H. Kersten, G. Thieme, R. Schneider, M. Hannemann, and R. Hippler, Rotating dust ring in an rf discharge coupled with a dc-magnetron sputter source. experiment and simulation, J. Phys. D: Appl. Phys. 37, 2703 (2004).

[8] A. E. Farsy, J. Ledig, M. Desecures, J. Bougdira, and L. de Poucques, Characterization of transport of titanium neutral atoms sputtered in ar and ar/n2 HIPIMS discharges, Plasma Sources Sci. Technol. 28, 035005 (2019).

[9] M. Cavarroc, M. Mikikian, Y. Tessier, and L. Boufendi, Successive generations of dust in complex plasmas: A cyclic phenomenon in the void region, Phys. Rev. Lett. 100, 045001 (2008).

[10] I. Stefanović, N. Sadeghi, and J. Winter, The influence of $\mathrm{C}_{2} \mathrm{H}_{2}$ and dust formation on the time dependence of metastable argon density in pulsed plasmas, J. Phys. D: Appl. Phys. 43, $152003(2010)$.

[11] D. Samsonov and J. Goree, Instabilities in a dusty plasma with ion drag and ionization, Phys. Rev. E 59, 1047 (1999).

[12] M. Mikikian, M. Cavarroc, L. Couëdel, and L. Boufendi, Low frequency instabilities during dust particle growth in a radio-frequency plasma, Phys. Plasmas 13, 092103 (2006).

[13] G. M. Jellum and D. B. Graves, Particulates in aluminum sputtering discharges, J. Appl. Phys. 67, 6490 (1990).

[14] C. Arnas, A. Michau, G. Lombardi, L. Couëdel, and K. K. Kumar, Effects of the growth and the charge of carbon nanoparticles on dc discharges, Phys. Plasmas 20, 013705 (2013).

[15] Kishor Kumar Kalathiparambil, L. Couëdel, and C. Arnas, Growth of tungsten nanoparticles in direct-current argon glow discharges, Phys. Plasmas 20, 043707 (2013).

[16] L. Couëdel, Kishor Kumar K., and C. Arnas, Detrapping of tungsten nanoparticles in a directcurrent argon glow discharge, Physics of Plasmas 21, 123703 (2014).

[17] S. Barbosa, L. Couëdel, C. Arnas, K. K. Kumar, C. Pardanaud, and F. R. A. Onofri, In-situ characterisation of the dynamics of a growing dust particle cloud in a direct-current argon glow discharge, J. Phys. D: Appl. Phys. 49, 045203 (2016).

[18] H. Haberland, M. Mall, M. Moseler, Y. Qiang, T. Reiners, and Y. Thurner, Filling of micronsized contact holes with copper by energetic cluster impact, J. Vac. Sci.Technol. A 12, 2925 
(1994).

[19] A. Majumdar, D. Köpp, M. Ganeva, D. Datta, S. Bhattacharyya, and R. Hippler, Development of metal nanocluster ion source based on dc magnetron plasma sputtering at room temperature, Rev. Sci. Instrum. 80, 095103 (2009).

[20] T. Momin and A. Bhowmick, A new magnetron based gas aggregation source of metal nanoclusters coupled to a double time-of-flight mass spectrometer system, Rev. Sci. Instrum. 81, $075110(2010)$.

[21] V. Bouchat, O. Feron, B. Gallez, B. Masereel, C. Michiels, T. V. Borght, and S. Lucas, Carbon nanoparticles synthesized by sputtering and gas condensation inside a nanocluster source of fixed dimension, Surf. Coat. Technol. 205, Supplement 2, S577 (2011), pSE 2010 Special Issue: Proceedings of the 12th International Conference on Plasma Surface Engineering.

[22] N. K. Manninen, N. M. Figueiredo, S. Carvalho, and A. Cavaleiro, Production and characterization of ag nanoclusters produced by plasma gas condensation, Plasma Processes Polym. 11, 629 (2014).

[23] M. V. Dutka, A. A. Turkin, D. I. Vainchtein, and J. T. M. De Hosson, On the formation of copper nanoparticles in nanocluster aggregation source, Journal of Vacuum Science \& Technology A 33, 031509 (2015).

[24] J. Kousal, A. Kolpaková, A. Shelemin, P. Kudrna, M. Tichý, O. Kylián, J. Hanuš, A. Choukourov, and H. Biederman, Monitoring of conditions inside gas aggregation cluster source during production of ti/tio x nanoparticles, Plasma Sources Sci. Technol. 26, 105003 (2017).

[25] T. Acsente, R. Negrea, L. Nistor, E. Matei, C. Grisolia, R. Birjega, and G. Dinescu, Tungsten nanoparticles with controlled shape and crystallinity obtained by magnetron sputtering and gas aggregation, Mater. Lett. 200, 121 (2017).

[26] M. Vaidulych, J. Hanuš, J. Kousal, S. Kadlec, A. Marek, I. Khalakhan, A. Shelemin, P. Solař, A. Choukourov, O. Kylián, and H. Biederman, Effect of magnetic field on the formation of $\mathrm{cu}$ nanoparticles during magnetron sputtering in the gas aggregation cluster source, Plasma Processes and Polymers 16, 1900133 (2019).

[27] M. Koten, S. Voeller, M. Patterson, and J. Shield, In situ measurements of plasma properties during gas-condensation of cu nanoparticles, J. Appl. Phys. 119, 114306 (2016).

[28] J. Kousal, A. Shelemin, M. Schwartzkopf, O. Polonskyi, J. Hanuš, P. Solař, M. Vaidulych, D. Nikitin, P. Pleskunov, Z. Krtouš, et al., Magnetron-sputtered copper nanoparticles: lost in 
gas aggregation and found by in situ x-ray scattering, Nanoscale 10, 18275 (2018).

[29] H. Hahn and R. S. Averback, The production of nanocrystalline powders by magnetron sputtering, J. Appl. Phys. 67, 1113 (1990).

[30] N. Nafarizal and K. Sasaki, Synthesis characteristics of cu particulates in high-pressure magnetron sputtering plasmas studied by in situ laser-light scattering, J. Phys. D: Appl. Phys. 45, $505202(2012)$.

[31] L. Couëdel, C. Arnas, T. Acsente, and A. Chami, Characterisation of a high-pressure directcurrent magnetron discharge used for tungsten nanoparticle production, AIP Conference Proceedings 1925, 020020 (2018).

[32] C. Arnas, A. Chami, L. Couëdel, T. Acsente, M. Cabié, and T. Neisius, Thermal balance of tungsten monocrystalline nanoparticles in high pressure magnetron discharges, Phys. Plasmas 26, 053706 (2019).

[33] A. Chami and C. Arnas, Spatial distributions of plasma parameters in conventional magnetron discharges in presence of nanoparticles, Journal of Plasma Physics 86, 905860512 (2020).

[34] S. Mitic, M. Y. Pustylnik, and G. E. Morfill, Spectroscopic evaluation of the effect of the microparticles on radiofrequency argon plasma, New J. Phys. 11, 083020 (2009).

[35] S. Mitic, B. Klumov, M. Pustylnik, and G. Morfill, Determination of electron temperature in low-pressure plasmas by means of optical emission spectroscopy, JETP Lett. 91, 231 (2010).

[36] S. M. Rossnagel and H. R. Kaufman, Current-voltage relations in magnetrons, Journal of Vacuum Science \& Technology A 6, 223 (1988).

[37] D. Depla, G. Buyle, J. Haemers, and R. De Gryse, Discharge voltage measurements during magnetron sputtering, Surface and Coatings Technology 200, 4329 (2006).

[38] G. Buyle, D. Depla, K. Eufinger, J. Haemers, R. D. Gryse, and W. D. Bosscher, Simplified model for calculating the pressure dependence of a direct current planar magnetron discharge, Journal of Vacuum Science \& Technology A: Vacuum, Surfaces, and Films 21, 1218 (2003).

[39] G. Buyle, D. Depla, K. Eufinger, J. Haemers, W. Bosscher, and R. Gryse, Simplified model for the $\{\mathrm{DC}\}$ planar magnetron discharge, Vacuum 74, 353 (2004), selected papers revised from the Proceedings of the Seventh International Symposium on Sputtering and Plasma Processes (ISSP 2003).

[40] R. L. Merlino, Understanding langmuir probe current-voltage characteristics, Am. J. Phys 75, 1078 (2007). 
[41] M. A. Lieberman and A. J. Lichtenberg, Principles of plasma discharges and materials processing (2nd edition), edited by J. W. . Sons (John Wiley \& Sons, 2005).

[42] The presence of small nanoparticle with diameter $\leq 10 \mathrm{~nm}$ can nevertheless not be ruled out.

[43] X.-M. Zhu and Y.-K. Pu, A simple collisional-radiative model for low-temperature argon discharge with pressure ranging from 1 pa to atmospheric pressure: kinetics of paschen 1s and 2p levels, J. Phys. D: Appl. Phys. 43, 015204 (2010).

[44] J. Kaupe, D. Coenen, and S. Mitic, Phase-resolved optical emission spectroscopy of a transient plasma created by a low-pressure dielectric barrier discharge jet, Plasma Sources Science and Technology 27, 105003 (2018).

[45] J. Kaupe, P. Riedl, D. Coenen, and S. Mitic, Temporal evolution of electron density and temperature in low pressure transient ar/n2 plasmas estimated by optical emission spectroscopy, Plasma Sources Science and Technology 28, 065012 (2019).

[46] S. Mitic, J. Kaupe, P. Riedl, and D. Coenen, Comparative studies of compact khz and mhz driven low pressure plasmas by emission and laser spectroscopy, Physics of Plasmas 26, 073507 (2019).

[47] T. Holstein, Imprisonment of resonance radiation in gases, Physical Review 72, 12 (1947).

[48] P. J. Walsh, Effect of simultaneous doppler and collisional broadening and a hyperfine structure on the imprisonment of resonance radiation, Physical Review 116, 3 (1959).

[49] M. Schulze, Yanguas-Gil, A. von Keudell, and P. Awakowitz, A robust method to measure metastable and resonant state densities from emission spectra in argon and argon-diluted low pressure plasmas, J. Phys. D: Appl. Phys. 41, 065206 (2008).

[50] R. Mewe, Relative intensity of helium spectral lines as a function of electron temperature and density, British Journal of Applied Physics 18, 107 (1967).

[51] NIST database, https://www.nist.gov (2020).

[52] NGFSRDW database, www.lxcat.net, retrieved on june 17, 2020. and BSR database, www.lxcat.net, retrieved on june 17, 2020. (2020).

[53] V. Gedeon, S. Gedeon, V. Lazur, E. Nagy, O. Zatsarinny, and K. Bartschat, $b$-spline $r$-matrixwith-pseudostates calculations for electron collisions with aluminum, Phys. Rev. A 92, 052701 (2015).

[54] J. Trieschmann, S. Ries, N. Bibinov, P. Awakowicz, S. Mráz, J. M. Schneider, and T. Mussenbrock, Combined experimental and theoretical description of direct current magnetron sput- 
tering of al by ar and ar/n2 plasma, Plasma Sources Sci. Technol. 27, 054003 (2018).

[55] K. Sasaki and N. Nafarizal, Enhancement of ti + density in high-pressure magnetron sputtering plasmas, J. Phys. D: Appl. Phys. 43, 124012 (2010).

[56] F. J. Jimenez and S. K. Dew, Comprehensive computer model for magnetron sputtering. i. gas heating and rarefaction, Journal of Vacuum Science \& Technology A 30, 041302 (2012).

[57] A. E. Wendt and M. A. Lieberman, Spatial structure of a planar magnetron discharge, Journal of Vacuum Science \& Technology A 8, 902 (1990).

[58] J. Hopwood and F. Qian, Mechanisms for highly ionized magnetron sputtering, J. Appl. Phys. 78, 758 (1995).

[59] C. Christou and Z. H. Barber, Ionization of sputtered material in a planar magnetron discharge, Journal of Vacuum Science \& Technology A: Vacuum, Surfaces, and Films 18, 2897 (2000).

[60] The plasma is approximated by an infinite cylinder.

[61] I. Stefanović, T. Kuschel, S. Schröter, and M. Böke, Argon metastable dynamics and lifetimes in a direct current microdischarge, Journal of Applied Physics 116, 113302 (2014), https://doi.org/10.1063/1.4895714.

[62] Cross sections extracted from program magboltz, version 7.1 june 2004, biagi-v7.1 database, www.lxcat.net, retrieved on april 20, 2021., https://www.lxcat.net/Biagi-v7.1 (2021). 